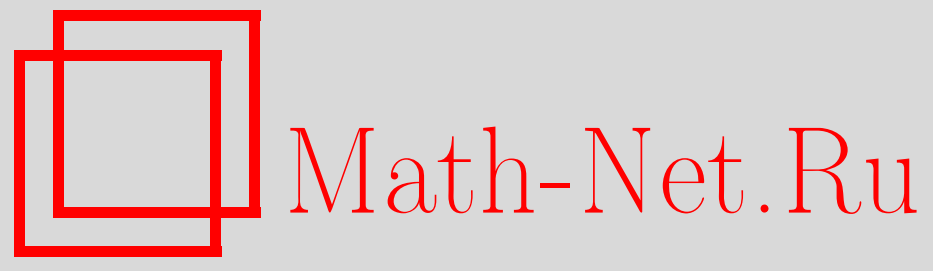

Ж. А. Балкизов, Задача со смещением для вырождающегося гиперболического уравнения первого рода, Вестн. Сам. гос. техн. ун-та. Сер. Физ.мат. науки, 2021, номер 1, 21-34

DOI: https://doi.org/10.14498/vsgtu1783

Использование Общероссийского математического портала MathNet.Ru подразумевает, что вы прочитали и согласны с пользовательским соглашением

http://www . mathnet.ru/rus/agreement

Параметры загрузки:

IP : 54.174 .149 .18

26 апреля 2023 г., 07:16:38

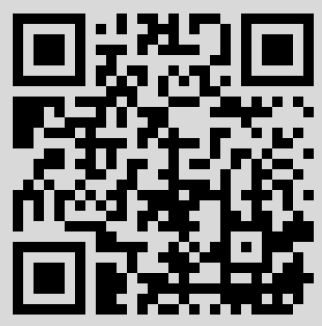


Вестн. Сам. гос. техн. ун-та. Сер. Физ.-мат. науки. 2021. Т. 25, № 1. С. 21-34 ISSN: 2310-7081 (online), 1991-8615 (print)

УДК 517.956.326

\title{
Задача со смещением для вырождающегося гиперболического уравнения первого рода
}

\author{
(C) Ж.А. Балкизов
}

Институт прикладной математики и автоматизации КБНЦ РАН, Россия, 360000, Нальчик, ул. Шортанова, 89 а.

\begin{abstract}
Аннотация
Рассматривается вырождающееся гиперболическое уравнение первого рода второго порядка, содержащее слагаемое с младшей производной, для которого исследованы две краевые задачи со смещением, обобщающие известные первую и вторую задачи Дарбу. При определенных условиях на заданные функции и параметры, входящие в постановку исследуемых задач, доказаны теоремы о существовании единственного регулярного решения задач. Выявлены свойства всех регулярных решений рассматриваемого уравнения, являющиеся аналогами теорем о среднем значении для волнового уравнения.
\end{abstract}

Ключевые слова: вырождающиеся гиперболические уравнения, задача Гурса, задача Дарбу, задача со смещением, теорема о среднем значении.

Получение: 20 апреля 2020 г. / Исправление: 12 февраля 2021 г. Принятие: 10 марта 2021 г. / Публикация онлайн: 29 марта 2021 г.

Введение. Постановка задач. На евклидовой плоскости точек $(x, y)$ рассмотрим уравнение

$$
(-y)^{m} u_{x x}-u_{y y}+\lambda(-y)^{\frac{m-2}{2}} u_{x}=0, \quad y<0,
$$

где $\lambda$ и $m$ - заданные действительные числа, причем $m>0,|\lambda| \leqslant m / 2$; $u=u(x, y)$ - искомая действительная функция действительных переменных $(x, y)$.

Уравнение (1) рассматривается в области $\Omega$, ограниченной его характеристиками

$$
A C: \quad x-\frac{2}{m+2}(-y)^{\frac{m+2}{2}}=0 \quad \text { и } \quad B C: \quad x+\frac{2}{m+2}(-y)^{\frac{m+2}{2}}=r,
$$

\section{Научная статья}

(2)(1) Контент публикуется на условиях лицензии Creative Commons Attribution 4.0 International (https://creativecommons.org/licenses/by/4.0/deed.ru)

\section{Образец для цитирования}

Бал к и зо в Ж. А. Задача со смещением для вырождающегося гиперболического уравнения первого рода // Вестн. Сам. гос. техн. ун-та. Сер. Физ.-мат. науки, 2021. Т. 25 , № 1. C. 21-34. https://doi.org/10.14498/vsgtu1783.

\section{Сведения об авторе}

Жираслан Анатольевич Балкизов (1) https://orcid.org/0000-0001-5329-7766

кандидат физико-математических наук; ведущий научный сотрудник; отд. уравнений смешанного типа; e-mail: giraslan@yandex.ru 
выходящими из точки $C=\left(r / 2, y_{c}\right), y_{c}=-\left[\frac{(m+2) r}{4}\right]^{2 /(m+2)}$ и проходящими через точки $A=(0,0)$ и $B=(r, 0)$ соответственно, а также отрезком $A B$ прямой $y=0$.

Обозначим через $\theta_{0}(x)=\left(\frac{x}{2}, h(x)\right), \theta_{r}(x)=\left(\frac{r+x}{2}, h(r-x)\right)$, где $h(x)=$ $=-\left(\frac{m+2}{4}\right)^{2 /(m+2)} x^{2 /(m+2)}$ - аффиксы точек пересечения характеристик уравнения $(1)$, выходящих из точки $(x, 0)$, с характеристиками $A C$ и $B C$ соответственно; $D_{0 x}^{\gamma} f(t)$ и $D_{x r}^{\gamma} f(t)$ - обобщенные операторы дробного (в смысле Римана-Лиувилля) интегро-дифференцирования порядка $\gamma[1,2]$;

$$
I=\{(x, y): 0<x<r, y=0\} ; \quad \alpha=\frac{m-2 \lambda}{2(m+2)}, \quad \beta=\frac{m+2 \lambda}{2(m+2)}
$$

Регулярным в области $\Omega$ решением уравнения (1) назовем всякую функцию $u=u(x, y)$ из класса $C(\bar{\Omega}) \cap C^{1}(\Omega \cup I) \cap C^{2}(\Omega)$, при подстановке которой уравнение (1) обращается в тождество.

ЗАДАчА 1. Найти регулярное в области $\Omega$ решение $u=u(x, y)$ уравнения (1) из класса $x^{-\alpha} u_{y}(x, 0),(r-x)^{-\beta} u_{y}(x, 0) \in L_{1}(I)$, удовлетворяющее краевым условиям

$$
\begin{gathered}
u(x, 0)=\tau(x) \quad \forall x \in \bar{I} \\
a(x) D_{0 x}^{1-\beta} u\left[\theta_{0}(t)\right]+b(x) D_{x r}^{1-\alpha} u\left[\theta_{r}(t)\right]=\psi(x) \quad \forall x \in I,
\end{gathered}
$$

где $a(x), b(x), \tau(x), \psi(x)$ - заданные функиии, причем $a^{2}(x)+b^{2}(x) \neq 0 \forall x \in \bar{I}$.

ЗАДАча 2. Найти регулярное в области $\Omega$ решение уравнения (1), удовлетворяюшее краевым условиям

$$
\begin{gathered}
u_{y}(x, 0)=\nu(x) \quad \forall x \in I, \\
a(x) D_{0 x}^{\alpha}\left\{t^{\alpha+\beta-1} u\left[\theta_{0}(t)\right]\right\}+b(x) D_{x r}^{\beta}\left\{(r-t)^{\alpha+\beta-1} u\left[\theta_{r}(t)\right]\right\}=\psi(x) \forall x \in I,
\end{gathered}
$$

где $a(x), b(x), \nu(x), \psi(x)$ - заданные функиии, причем $a^{2}(x)+b^{2}(x) \neq 0 \forall x \in \bar{I}$.

Уравнение (1) является уравнением гиперболического типа с параболическим вырождением вдоль прямой $y=0$, причем прямая $y=0$ сама не является характеристикой уравнения (1). Поэтому уравнение (1) относится к классу вырождающихся гиперболических уравнений первого рода [3, с. 6].

При $a(x) \equiv 0, b(x) \neq 0 \forall x \in \bar{I}$ или $b(x) \equiv 0, a(x) \neq 0 \forall x \in \bar{I}$ из задачи 1 в силу обратимости операторов $D_{0 x}^{\gamma}$ и $D_{x r}^{\gamma}$ приходим к соответствующей первой задаче Дарбу с данными $u(x, 0)=\tau(x), u\left[\theta_{r}(x)\right]=\psi_{1}(x)$ или $u(x, 0)=\tau(x), u\left[\theta_{0}(x)\right]=\psi_{1}(x)$ для уравнения (1). А задача 2 при $a(x) \equiv 0$, $b(x) \neq 0 \forall x \in \bar{I}$ или $b(x) \equiv 0, a(x) \neq 0 \forall x \in \bar{I}$ переходит во вторую задачу Дарбу для уравнения (1), где заданы

$$
u_{y}(x, 0)=\nu(x),\left.\quad u\right|_{B C}=u\left[\theta_{r}(x)\right]=\psi_{1}(x), \quad 0<x<r
$$

или

$$
u_{y}(x, 0)=\nu(x),\left.\quad u\right|_{A C}=u\left[\theta_{0}(x)\right]=\psi_{1}(x), \quad 0<x<r,
$$

соответственно. 
При $m=2$ уравнение (1) переходит в уравнение Бицадзе-Лыкова [4, с. 47; 5; 6, с. 234]. В работе [7] показано, что для уравнения Бицадзе-Лыкова, которое рассматривается в области $D$, ограниченной характеристиками $A C$ : $2 x-y^{2}=0, B C: 2 x+y^{2}=2 r$ и отрезком $I$, при $\lambda=1$ корректно поставлена вторая задача Дарбу с данными (7), в то время как однородная вторая задача Дарбу для уравнения Бицадзе-Лыкова, соответствующая задаче (6), имеет ненулевые решения. Аналогично, для уравнения Бицадзе-Лыкова при $\lambda=-1$ будет корректно поставлена вторая задача Дарбу с данными (6), в то время как однородная вторая задача Дарбу, соответствующая задаче (7), будет обладать ненулевыми решениями. Это говорит о неравноправности характеристик $A C$ и $B C$ как носителей второй задачи Дарбу для уравнения Бицадзе-Лыкова при $\lambda= \pm 1$.

Частным случаем уравнения (1) также является уравнение Трикоми, являющееся теоретической основой околозвуковой газовой динамики и математической биологии [8; 9, с. 38; 10 с. 280].

При $\lambda=0$ из уравнения (1) приходим к уравнению Геллерстедта

$$
(-y)^{m} u_{x x}-u_{y y}=0
$$

которое, как показано в монографии [2, с. 234], находит применение в задаче определения формы прорези плотины.

Основным отличием уравнений (1) и (8) является тот факт, что для уравнения (1) при $\lambda \neq 0$ нарушено условие Геллерстедта [11], гарантирующее однозначную разрешимость задачи Коши для класса вырождающихся гиперболических уравнений с начальными данными на линии вырождения. Но упомянутое условие Геллерстедта, гарантирующее корректность задачи Коши, не является необходимым для ее однозначной разрешимости. И в монографиях $[3,4,6]$ уравнение (1) приводится как пример уравнения, для которого при $|\lambda| \leqslant m / 2$ решение задачи Коши выписывается в замкнутом виде, несмотря на то, что для него нарушено условие Геллерстедта при $\lambda \neq 0$. Исследованию первой и второй задач Дарбу для уравнения (1) посвящены работы [12, 13]. В работе [14] исследован критерий непрерывности решения задачи Гурса для вырождающегося гиперболического уравнения вида (1). В [15] в явном виде выписано регулярное решение задачи Гурса для вырождающегося внутри области гиперболического уравнения, а в [16] найдено решение первой краевой задачи для такого же уравнения. За последние годы многими авторами изучены задачи для разного рода вырождающихся гиперболических уравнений [17-28]. Достаточно полная библиография по исследованию различных краевых задач для вырождающихся гиперболических уравнений имеется в монографиях [29-34].

Исследуемые в рамках данной работы задачи 1 и 2 относятся к классу нелокальных краевых задач, сформулированных в работе [35], где дана методика постановки нелокальных краевых задач со смещением для вырождающегося гиперболического уравнения вида (8) с использованием понятия оператора дробного интегро-дифференцирования (в смысле РиманаЛиувилля). В [35] были найдены критерии однозначной разрешимости задачи с условиями вида (2) и (3) для рассматриваемого уравнения, где $\theta_{0}(x), \theta_{r}(x)$, как и выше, определяются как аффиксы точек пересечения характеристик уравнения (8), причем здесь $\alpha=\beta=m /[2(m+2)]=\varepsilon$. Также в [35] было получено свойство всех регулярных решений уравнения (8), удовлетворяющих 
начальному условию (2), являющееся аналогом теоремы о среднем значении для волнового уравнения в характеристическом четырехугольнике.

Пользуясь методом, предложенным в работе [35], в данной работе найдены достаточные условия существования единственного регулярного решения исследуемых задач 1 и 2 . Получены свойства регулярных решений уравнения (1), удовлетворяющих начальным условиям (2) или (4). Полученные свойства обобщают известные теоремы о среднем значении для уравнения (8) [35] и для волнового уравнения [8, с. 165] в характеристическом четырехугольнике.

\section{1. Исследование задачи 1. Справедлива следующая}

Теорема 1. Пусть заданные функиии $a(x), b(x), \tau(x), \psi(x)$ таковы, что

$$
\begin{gathered}
D_{0 x}^{-\alpha-\beta} \tau(t), D_{x r}^{-\alpha-\beta} \tau(t) \in C^{1}(\bar{I}) \cup C^{3}(I) ; \\
a(x), b(x), \psi(x) \in C(\bar{I}) \cap C^{2}(I) ; \\
\Gamma(1-\beta) a(x)(r-x)^{\beta}+\Gamma(1-\alpha) b(x) x^{\alpha} \neq 0 \forall x \in \bar{I} .
\end{gathered}
$$

Тогда существует единственное регулярное в области $\Omega$ решение задачи 1.

До ка з а т е ль с т в о. Воспользуемся представлением решения задачи Коши для уравнения (1) с данными $u(x, 0)=\tau(x)$ и $u_{y}(x, 0)=\nu(x)$ на линии вырождения $y=0$. Для различных значений числа $\lambda$ данные представления выписаны, например, в [3, с. 13] и они имеют вид

$$
\begin{array}{r}
u(x, y)=\frac{\Gamma(\alpha+\beta)}{\Gamma(\alpha) \Gamma(\beta)} \int_{0}^{1} \tau\left[x+\frac{2}{m+2}(-y)^{\frac{m+2}{2}}(2 t-1)\right] t^{\beta-1}(1-t)^{\alpha-1} d t+ \\
+\frac{\Gamma(2-\alpha-\beta) y}{\Gamma(1-\alpha) \Gamma(1-\beta)} \int_{0}^{1} \nu\left[x+\frac{2}{m+2}(-y)^{\frac{m+2}{2}}(2 t-1)\right] t^{-\alpha}(1-t)^{-\beta} d t \\
-\frac{m}{2}<\lambda<\frac{m}{2}
\end{array}
$$

$$
\begin{aligned}
& u(x, y)=\tau\left(x-\frac{2}{m+2}(-y)^{\frac{m+2}{2}}\right)+ \\
& +\frac{2 y}{m+2} \int_{0}^{1} \nu\left[x-\frac{2}{m+2}(-y)^{\frac{m+2}{2}}(2 t-1)\right](1-t)^{-\frac{m}{m+2}} d t, \quad \lambda=-\frac{m}{2} ; \\
& u(x, y)=\tau\left(x+\frac{2}{m+2}(-y)^{\frac{m+2}{2}}\right)+ \\
& \quad+\frac{2 y}{m+2} \int_{0}^{1} \nu\left[x+\frac{2}{m+2}(-y)^{\frac{m+2}{2}}(2 t-1)\right](1-t)^{-\frac{m}{m+2}} d t, \quad \lambda=\frac{m}{2} .
\end{aligned}
$$

Рассмотрим сначала случай, когда $-m / 2<\lambda<m / 2$. Из представления (12) в этом случае находим

$$
\begin{aligned}
u\left[\theta_{0}(x)\right]=u & \left(\frac{x}{2},-\left(\frac{m+2}{4}\right)^{\frac{2}{m+2}} x^{\frac{2}{m+2}}\right)=\frac{\Gamma(\alpha+\beta)}{\Gamma(\alpha) \Gamma(\beta)} \int_{0}^{1} \tau(x t) t^{\beta-1}(1-t)^{\alpha-1} d t- \\
& -\frac{\Gamma(2-\alpha-\beta)}{\Gamma(1-\alpha) \Gamma(1-\beta)}\left(\frac{4}{m+2}\right)^{-\frac{2}{m+2}} x^{\frac{2}{m+2}} \int_{0}^{1} \nu(x t) t^{-\alpha}(1-t)^{\beta} d t .
\end{aligned}
$$


После введения новой переменной интегрирования $z=x t$ последнее равенство перепишется в виде

$$
\begin{aligned}
u\left[\theta_{0}(x)\right]=\frac{\Gamma(\alpha+\beta)}{\Gamma(\alpha) \Gamma(\beta)} & x^{1-\alpha-\beta} \int_{0}^{x} \frac{\tau(z) z^{\beta-1}}{(x-z)^{1-\alpha}} d z- \\
& -\frac{\Gamma(2-\alpha-\beta)}{\Gamma(1-\alpha) \Gamma(1-\beta)}[2(1-\alpha-\beta)]^{\alpha+\beta-1} \int_{0}^{x} \frac{z^{-\alpha} \nu(z)}{(x-z)^{\beta}} d z .
\end{aligned}
$$

Воспользовавшись далее определением оператора интегрирования дробного порядка

$$
\int_{0}^{x} \frac{g(t) d t}{(x-t)^{1-\alpha}}=\Gamma(\alpha) D_{0 x}^{-\alpha} g(t)
$$

находим

$$
u\left[\theta_{0}(x)\right]=\gamma_{1} x^{1-\alpha-\beta} D_{0 x}^{-\alpha}\left[t^{\beta-1} \tau(t)\right]-\gamma_{2} D_{0 x}^{\beta-1}\left[t^{-\alpha} \nu(t)\right]
$$

где

$$
\gamma_{1}=\frac{\Gamma(\alpha+\beta)}{\Gamma(\beta)}, \quad \gamma_{2}=\frac{\Gamma(2-\alpha-\beta)}{\Gamma(1-\alpha)}[2(1-\alpha-\beta)]^{\alpha+\beta-1} .
$$

С помощью аналогичных вычислений из формулы (12) получаем, что

$$
u\left[\theta_{r}(x)\right]=\gamma_{3}(r-x)^{1-\alpha-\beta} D_{x r}^{-\beta}\left[(r-t)^{\alpha-1} \tau(t)\right]-\gamma_{4} D_{x r}^{\alpha-1}\left[(r-t)^{-\beta} \nu(t)\right],
$$

где

$$
\gamma_{3}=\frac{\Gamma(\alpha+\beta)}{\Gamma(\alpha)}, \quad \gamma_{4}=\frac{\Gamma(2-\alpha-\beta)}{\Gamma(1-\beta)}[2(1-\alpha-\beta)]^{\alpha+\beta-1} .
$$

Воспользовавшись следующими законами композиции операторов дробного дифференцирования и интегрирования [1; 2, с. 18]:

$$
\begin{gathered}
D_{0 x}^{\alpha} t^{\alpha+\beta} D_{0 t}^{\beta} g(s)=x^{\beta} D_{0 x}^{\alpha+\beta} t^{\alpha} g(t), \\
D_{x r}^{\alpha}(r-t)^{\alpha+\beta} D_{t r}^{\beta} g(s)=(r-x)^{\beta} D_{x r}^{\alpha+\beta}(r-t)^{\alpha} g(t),
\end{gathered}
$$

из (15) и (16) находим

$$
\begin{gathered}
D_{0 x}^{1-\beta} u\left[\theta_{0}(t)\right]=x^{-\alpha}\left[\gamma_{1} D_{0 x}^{1-\alpha-\beta} \tau(t)-\gamma_{2} \nu(x)\right] \\
D_{x r}^{1-\alpha} u\left[\theta_{r}(t)\right]=(r-x)^{-\beta}\left[\gamma_{3} D_{x r}^{1-\alpha-\beta} \tau(t)-\gamma_{4} \nu(x)\right]
\end{gathered}
$$

Подставляя значения $D_{0 x}^{1-\beta} u\left[\theta_{0}(t)\right]$ и $D_{x r}^{1-\alpha} u\left[\theta_{r}(t)\right]$ из (17) и (18) в граничное условие (3), будем иметь

$$
\begin{aligned}
a(x) x^{-\alpha}\left[\gamma_{1} D_{0 x}^{1-\alpha-\beta} \tau(t)-\right. & \left.\gamma_{2} \nu(x)\right]+ \\
& +b(x)(r-x)^{-\beta}\left[\gamma_{3} D_{x r}^{1-\alpha-\beta} \tau(t)-\gamma_{4} \nu(x)\right]=\psi(x),
\end{aligned}
$$

откуда

$$
\begin{aligned}
& {\left[\gamma_{2} a(x)(r-x)^{\beta}+\gamma_{4} b(x) x^{\alpha}\right] \nu(x)=} \\
& =\gamma_{1} a(x)(r-x)^{\beta} D_{0 x}^{1-\alpha-\beta} \tau(t)+\gamma_{3} b(x) x^{\alpha} D_{x r}^{1-\alpha-\beta} \tau(t)-x^{\alpha}(r-x)^{\beta} \psi(x)
\end{aligned}
$$


Если для всех $x \in \bar{I}$ выполнено условие (11) теоремы 1, то из (19) можно однозначно определить функцию $u_{y}(x, 0)=\nu(x)$. Тогда единственное регулярное решение задачи 1 будет выписываться по формуле (12). При $\lambda=-m / 2$ подобные рассуждения с использованием формулы (13) вновь приводят к уравнению вида (19) относительно функции $u_{y}(x, 0)=\nu(x)$, но при $\alpha=m /(m+2)$ : $\beta=0, \gamma_{1}=0, \gamma_{2}=2^{\alpha-1}(1-\alpha)^{\alpha}, \gamma_{3}=1, \gamma_{4}=2^{\alpha-1}(1-\alpha)^{\alpha} \Gamma(1-\alpha)$, а при $\lambda=m / 2: \alpha=0, \beta=m /(m+2), \gamma_{1}=1, \gamma_{2}=\Gamma(1-\beta) 2^{\beta-1}(1-\beta)^{\beta}, \gamma_{3}=0$, $\gamma_{4}=2^{\beta-1}(1-\beta)^{\beta}$. И в том и в другом случае после нахождения функции $u_{y}(x, 0)=\nu(x)$ решение задачи 1 выписывается по соответствующей формуле (13) или (14). Условия (9), (10) на заданные функции обеспечивают регулярность полученного решения.

Пусть теперь для всех $x \in \bar{I}$ нарушено условие (11) теоремы 1 , то есть пусть

$$
\Gamma(1-\beta) a(x)(r-x)^{\beta}+\Gamma(1-\alpha) b(x) x^{\alpha} \equiv 0 \quad \forall x \in \bar{I} .
$$

В этом случае из (3) и (19) после простых вычислений приходим к равенству

$$
\begin{aligned}
& \sin (\pi \beta)\left[\Gamma(\beta) x^{\alpha} D_{0 x}^{1-\beta} u\left[\theta_{0}(t)\right]-\Gamma(\alpha+\beta) D_{0 x}^{1-\alpha-\beta} \tau(t)\right]= \\
& \quad=\sin (\pi \alpha)\left[\Gamma(\alpha)(r-x)^{\beta} D_{x r}^{1-\alpha} u\left[\theta_{r}(t)\right]-\Gamma(\alpha+\beta) D_{x r}^{1-\alpha-\beta} \tau(t)\right]
\end{aligned}
$$

Формула (20) выражает свойство всех регулярных решений уравнения (1), удовлетворяющих условию (2), и является одним из аналогов теоремы о среднем значении для волнового уравнения [8, с. 165]. В частном случае уравнения $(1)$, когда $\lambda=0, \alpha=\beta=m /[2(m+2)]$, формула (20) получена в работе [35].

\section{2. Исследование задачи 2. Справедлива следующая}

Теорема 2. Пусть заданнъе функиии $a(x), b(x), \nu(x), \psi(x)$ таковы, что они обладают свойствами

$$
\begin{aligned}
& a(x), b(x), \quad \psi(x) \in C(\bar{I}) \cap C^{2}(I) ; \\
& \nu(x) \in C^{2}(I) ; \quad x^{-\alpha} \nu(x),(r-x)^{-\beta} \nu(x) \in L_{1}(I) \\
& \Gamma(\alpha) a(x)(r-x)^{1-\alpha}+\Gamma(\beta) b(x) x^{1-\beta} \neq 0 \quad \forall x \in \bar{I} \\
& \text { при } a(x) b(x) \neq 0 \quad \forall x \in \bar{I} \text {; } \\
& \lambda \neq \frac{m}{2} \text { nрu } a(x) \equiv 0 \quad \forall x \in \bar{I} \text {; } \\
& \lambda \neq-\frac{m}{2} \quad n p u \quad b(x) \equiv 0 \quad \forall x \in \bar{I} .
\end{aligned}
$$

Тогда существует единственное решение задачи 2.

Доказательство. Как и при доказательстве теоремы 1, из формул (12), (15), (16) находим, что

$$
\begin{aligned}
D_{0 x}^{\alpha}\left\{t^{\alpha+\beta-1} u\left[\theta_{0}(t)\right]\right\} & =x^{\beta-1}\left[\gamma_{1} \tau(x)-\gamma_{2} D_{0 x}^{\alpha+\beta-1} \nu(t)\right], \\
D_{x r}^{\beta}\left\{(r-t)^{\alpha+\beta-1} u\left[\theta_{r}(t)\right]\right\} & =(r-x)^{\alpha-1}\left[\gamma_{3} \tau(x)-\gamma_{4} D_{x r}^{\alpha+\beta-1} \nu(t)\right] .
\end{aligned}
$$


Подставляя значения $D_{0 x}^{\alpha}\left\{t^{\alpha+\beta-1} u\left[\theta_{0}(t)\right]\right\}$ и $D_{x r}^{\beta}\left\{(r-t)^{\alpha+\beta-1} u\left[\theta_{r}(t)\right]\right\}$ из (26) и $(27)$ в граничное условие $(5)$, будем иметь

$$
\begin{aligned}
& {\left[\gamma_{1} a(x)(r-x)^{1-\alpha}+\gamma_{3} b(x) x^{1-\beta}\right] \tau(x)=\psi(x)(r-x)^{1-\alpha} x^{1-\beta}+} \\
& \quad+\gamma_{2} a(x)(r-x)^{1-\alpha} D_{0 x}^{\alpha+\beta-1} \nu(t)+\gamma_{4} b(x) x^{1-\beta} D_{x r}^{\alpha+\beta-1} \nu(t)
\end{aligned}
$$

При условиях (23), (24), (25) из (28) однозначно определяется искомая функция $u(x, 0)=\tau(x)$. Тогда единственное регулярное решение задачи 2 в зависимости от значений $|\lambda|<m / 2, \lambda=-m / 2$ или $\lambda=m / 2$ будет выписываться по одной из формул (12), (13) или (14) соответственно. Условия (21), (22) обеспечивают регулярность полученного решения.

Пусть далее для всех $x \in \bar{I}$ нарушено условие (23), то есть пусть

$$
\Gamma(\alpha) a(x)(r-x)^{1-\alpha}+\Gamma(\beta) b(x) x^{1-\beta} \equiv 0 \quad \forall x \in \bar{I}
$$

при $a(x) b(x) \neq 0 \forall x \in \bar{I}$.

Тогда из граничного условия (5) и соотношения (28) приходим к равенству

$$
\begin{gathered}
\Gamma(\beta) x^{1-\beta} D_{0 x}^{\alpha}\left[t^{\alpha+\beta-1} u\left[\theta_{0}(t)\right]\right]-\Gamma(\alpha)(r-x)^{1-\alpha} D_{r x}^{\beta}\left[(r-t)^{\alpha+\beta-1} u\left[\theta_{r}(t)\right]\right]= \\
=\gamma_{5}\left[\Gamma(\beta) \Gamma(1-\beta) D_{0 x}^{\alpha+\beta-1} \nu(t)-\Gamma(\alpha) \Gamma(1-\alpha) D_{r x}^{\alpha+\beta-1} \nu(t)\right],
\end{gathered}
$$

где

$$
\gamma_{5}=-\frac{[2(1-\alpha-\beta)]^{\alpha+\beta-1}}{B(1-\alpha, 1-\beta)},
$$

$B(p, q)$ - бета-функция.

Равенство (29), так же как и (20), выражает один из аналогов теоремы о среднем значении для волнового уравнения, которым обладают все регулярные решения уравнения (1), удовлетворяющие условию (4).

3. Неравноправие характеристик как носителей данных задачи 2. Выше было отмечено, что задача 2 при $a(x) \equiv 0, b(x) \neq 0 \forall x \in \bar{I}$ или $b(x) \equiv 0$, $a(x) \neq 0 \forall x \in \bar{I}$ переходит во вторую задачу Дарбу для уравнения (1). Покажем, что при $\lambda= \pm m / 2$ характеристики $A C$ и $B C$, ограничивающие область $\Omega$, являются неравноправными как носители данных задачи 2 и из разрешимости задачи 2 с данными на одной из характеристик, вообще говоря, не следует разрешимость этой задачи с данными на другой характеристике.

Пусть для всех $x \in \bar{I}$ нарушено условие $(24)$, то есть

$$
\lambda=\frac{m}{2} \text { и } a(x) \equiv 0, b(x) \neq 0 \quad \forall x \in \bar{I}
$$

и пусть $D_{x r}^{-\beta}\left[\frac{\psi(t)(r-t)}{b(t)}\right] \in C^{1}(\bar{I}) \cup C^{3}(I)$. В этом случае однородная задача, соответствующая исследуемой задаче $2(\nu(x) \equiv 0, \psi(x) \equiv 0)$, обладает ненулевыми решениями вида

$$
u(x, y)=g\left(x+\frac{2}{m+2}(-y)^{\frac{m+2}{2}}\right),
$$


где $g(x) \in C^{1}(\bar{I}) \cap C^{2}(I)$ - произвольная функция, а сама неоднородная задача 2 разрешима тогда и только тогда, когда относительно заданных функций $b(x), \nu(x)$ и $\psi(x)$ выполнено дополнительное условие согласования

$$
\nu(x)=-\frac{1}{\gamma_{4}} D_{x r}^{1-\beta}\left[\frac{\psi(t)(r-t)}{b(t)}\right]=\psi_{*}(x) .
$$

Если условие (30) выполнено, то совокупность всех решений задачи 2 будет выражаться по формуле

$$
\begin{aligned}
u(x, y)=g(x+ & \left.\frac{2}{m+2}(-y)^{\frac{m+2}{2}}\right)+ \\
& +\frac{2 y}{m+2} \int_{0}^{1} \psi_{*}\left[x+\frac{2}{m+2}(-y)^{\frac{m+2}{2}}(2 t-1)\right](1-t)^{-\frac{m}{m+2}} d t,
\end{aligned}
$$

где, как и выше, $g(x)$ - произвольная функция из класса $C^{1}(\bar{I}) \cap C^{2}(I)$, а $\psi_{*}(x)$ определяется из формулы (30).

В то же время отметим, что задача 2 в случае, когда

$$
\lambda=m / 2, b(x) \equiv 0, a(x) \neq 0 \quad \forall x \in \bar{I},
$$

имеет единственное решение, которое выписывается по формуле (14), где

$$
\tau(x)=\frac{\psi(x) x^{1-\beta}}{a(x)}+\gamma_{2} D_{0 x}^{\beta-1} \nu(t) .
$$

Аналогично, если для всех $x \in \bar{I}$ нарушено условие (25), то есть если

$$
\lambda=-m / 2, b(x) \equiv 0, a(x) \neq 0 \quad \forall x \in \bar{I},
$$

и заданные функции $a(t), \psi(t)$ обладают свойствами

$$
D_{0 x}^{-\alpha}\left[\frac{\psi(t) t}{a(t)}\right] \in C^{1}(\bar{I}) \cup C^{3}(I),
$$

то в этом случае однородная задача, соответствующая исследуемой задаче 2 , будет иметь нетривиальные решения вида

$$
u(x, y)=g\left(x-\frac{2}{m+2}(-y)^{\frac{m+2}{2}}\right)
$$

где $g(x) \in C^{1}(\bar{I}) \cap C^{2}(I)$ - произвольная функция, а задача 2 будет разрешима тогда и только тогда, когда относительно заданных функций $a(x), \nu(x)$ и $\psi(x)$ выполнено условие согласования вида

$$
\nu(x)=-\frac{1}{\gamma_{2}} D_{0 x}^{1-\alpha}\left[\frac{\psi(t) t}{a(t)}\right]=\psi_{*}(x) .
$$

Если условие (31) выполнено, то множество решений задачи 2 будет выражаться по формуле 


$$
\begin{aligned}
u(x, y)=g(x- & \left.\frac{2}{m+2}(-y)^{\frac{m+2}{2}}\right)+ \\
& +\frac{2 y}{m+2} \int_{0}^{1} \psi_{*}\left[x-\frac{2}{m+2}(-y)^{\frac{m+2}{2}}(2 t-1)\right](1-t)^{-\frac{m}{m+2}} d t .
\end{aligned}
$$

Если же

$$
\lambda=-m / 2, a(x) \equiv 0, b(x) \neq 0 \quad \forall x \in \bar{I},
$$

то задача 2 однозначно разрешима и его единственное решение выписывается по формуле (13), где

$$
\tau(x)=\frac{\psi(x)(r-x)^{1-\alpha}}{b(x)}+\gamma_{4} D_{x r}^{\alpha-1} \nu(t) .
$$

Приведенные выше рассуждения еще раз подтверждают эффект неравноправия характеристик $A C$ и $B C$ как носителей данных второй задачи Дарбу для уравнения (1), полученный А. М. Нахушевым в работе [7] в случае, когда $m=2$ и $\lambda= \pm 1$.

Заключение. В работе исследованы две нелокальные краевые задачи со смещением для вырождающегося гиперболического уравнения первого рода второго порядка вида (1), являющиеся обобщением обычных первой и второй задач Дарбу для таких уравнений.

Найдены достаточные условия существования единственного регулярного решения исследуемых задач. Получены равенства, выражающие свойства всех регулярных решений рассматриваемого уравнения (1), удовлетворяющие либо начальному условию $u(x, 0)=\tau(x)$, либо условию $u_{y}(x, 0)=\nu(x)$.

Полученные свойства являются аналогами теорем о среднем значении для волнового уравнения и найдут применение при дальнейших исследованиях различных локальных и нелокальных краевых задач для вырождающихся гиперболических уравнений и уравнений смешанного типа с вырождающимся гиперболическим оператором вида (1) в главной части.

Конкурирующие интересы. Я заявляю об отсутствии явных и потенциальных конфликтов интересов, связанных с публикацией настоящей статьи.

Авторский вклад и ответственность. Я несу полную ответственность за предоставление окончательной версии рукописи в печать. Окончательная версия рукописи мною одобрена

Финансирование. Исследование выполнялось без финансирования.

\section{Библиографический список}

1. Самко С. Г., Килбас А. А., Маричев О. И. Интегралы и производные дробного порядка и некоторые их приложения. Минск: Наука и техника, 1987. 688 с.

2. Нахушев А. М. Дробное исчисление и его применение. М.: Физматлит, 2003. 272 с.

3. Смирнов М. М. Вырождающиеся гиперболические уравнения. Минск: Выш. шк., 1977. $160 \mathrm{c}$.

4. Бицадзе А. В. Уравнения смешанного типа. М.: АН СССР, 1959. 164 с.

5. Лыков А. В. Применение методов термодинамики необратимых процессов к исследованию тепло и массообмена // Инж.-физ. ж., 1965. Т. 9, № 3. С. 287-304.

6. Бицадзе А. В. Некоторые классы уравнений в частных производных. М.: Наука, 1981. $448 \mathrm{c}$. 
7. Нахушев А. М. О задаче Дарбу для вырождающихся гиперболических уравнений // Диффер. уравн., 1971. Т. 7, №1. С. 49-56.

8. Нахушев А. М. Уравнения математической биологии. М.: Высш. шк., 1995. 301 с.

9. Берс Л. Математические вопросы дозвуковой и околозвуковой газовой динамики. М.: Иностр. литер., 1961. 208 с.

10. Франкль Ф. И. Избранные труды по газовой динамике. М.: Наука, 1973. 711 с.

11. Gellerstedt S. Sur une équation linéaire aux dérivées partielles de type mixte// Ark. Mat. Astron. Fys. A, 1937. vol. 25, no. 29. pp. 1-23.

12. Кальменов Т. Ш. Критерий единственности решения задачи Дарбу для одного вырождающегося гиперболического уравнения // Диффер. уравн., 1971. Т. 7, № 1. С. 178-181.

13. Кальменов Т. Ш. О задаче Дарбу для одного вырождающегося уравнения // Диффер. уравн., 1974. Т. 10, № 1. С. 59-68.

14. Кальменов Т. Ш. Критерий непрерывности решения задачи Гурса для одного вырождающегося уравнения // Дифбер. уравн., 1972. Т. 8, № 1. С. 41-54.

15. Балкизов Ж. А. Краевая задача для вырождающегося внутри области гиперболического уравнения // Изв. вуз. Северо-Кавказ. регион. Сер. Естеств. науки, 2016. № 1(189). C. 5-10. https://doi.org/10.18522/0321-3005-2016-1-5-10.

16. Балкизов Ж. А. Первая краевая задача для вырождающегося внутри области гиперболического уравнения // Владикавк. матем. журн., 2016. Т. 18, № 2. С. 19-30.

17. Кириченко С. В. Смешанная задача с интегральным условием для вырождающегося уравнения гиперболического типа // Вестн. СамГУ. Естественнонаучн. сер., 2011. T. 17, № 8. C. 29-36. https://doi .org/10.18287/2541-7525-2011-17-8-29-36.

18. Репин О. А., Кумыкова С. К. О задаче с обобщенными операторами дробного дифференцирования для вырождающегося внутри области гиперболического уравнения // Вестн. СамГУ. Естественнонаучн. сер., 2012. Т. 18, №9. С. 52-60. https://doi.org/ 10.18287/2541-7525-2012-18-9-52-60.

19. Репин О. А., Кумыкова С. К. Задача со смещением для вырождающегося внутри области гиперболического уравнения // Вестн. Сам. гос. техн. ун-та. Сер. Физ.-мат. науки, 2014. №1(34). C. 37-47. https://doi.org/10.14498/vsgtu1280.

20. Репин О. А., Кумыкова С. К. Об одном классе нелокальных задач для гиперболического уравнения с вырождением типа и порядка // Вестн. Сам. гос. техн. ун-та. Сер. Физ.-мат. науки, 2014. № 4(37). C. 22-32. https://doi.org/10.14498/vsgtu1348.

21. Эргашев Т. Г. Обобщенные решения одного вырождающегося гиперболического уравнения второго рода со спектральным параметром // Вестн. Томск. гос. ун-та. Матем. u мех., 2017. № 46. C. 41-49. https://doi.org/10.17223/19988621/46/6.

22. Макаова Р. Х. Краевая задача для гиперболического уравнения третьего порядка с вырождением порядка внутри области // Вестн. Сам. гос. техн. ун-та. Сер. Физ.-мат. науки, 2017. T. 21, № 4. C. 651-664. https://doi.org/10.14498/vsgtu1574.

23. Sabitov K. B., Zaitseva N. V. Initial-boundary value problem for hyperbolic equation with singular coefficient and integral condition of second kind// Lobachevskii J. Math., 2018. vol. 39, no. 9. pp. 1419-1427. https://doi.org/10.1134/S1995080218090299.

24. Сабитов К. Б., Зайцева Н. В. Вторая начально-граничная задача для $B$-гиперболического уравнения // Изв. вузов. Матем., 2019. №10. С. 75-86. https://doi.org/10. 26907/0021-3446-2019-10-75-86.

25. Сабитов К. Б., Сидоров С. Н. Начально-граничная задача для неоднородных вырождающихся уравнений смешанного параболо-гиперболического типа / Дифференциальные уравнения. Математическая физика/ Итоги науки и техн. Сер. Соврем. мат. и ее прил. Темат. обз., Т. 137. Москва: ВИНИТИ РАН, 2017. С. 26-60.

26. Уринов А. К., Окбоев А. Б. Видоизмененная задача Коши для одного вырождающегося гиперболического уравнения второго рода // Укр. матем. журн., 2020. Т. 72, № 1 . C. $100-118$. 
27. Макаова Р. Х. Краевая задача для вырождающегося внутри области гиперболического уравнения третьего порядка с оператором Аллера в главной части / Материаль международной научной конферениии «Актуальные проблемы прикладной математики и физики» (Кабардино-Балкария, Нальчик, 17-21 мая 2017 г.) / Итоги науки и техн. Сер. Соврем. мат. и ее прил. Темат. обз., Т. 149. М.: ВИНИТИ РАН, 2018. С. 64-71.

28. Кожанов А. И. Начально-граничные задачи для вырождающихся гиперболических уравнений// Сиб. электрон. матем. изв., 2021. Т.18. С. 43-53. https://doi.org/ 10.33048/semi.2021.18.004.

29. Нахушев А. М. Об одном классе линейных краевых задач для гиперболического и смешанного типов уравнений второго порлдка. Нальчик: Эльбрус, 1992. 155 с.

30. Репин О. А. Краевые задачи со смещением для уравнений гиперболического и смешанного типов. Самара: Саратовск. гос. унив., Самарск. фил., 1992. 164 с.

31. Кальменов Т. Ш. Краевые задачи для линейных уравнений в частных производных гиперболического типа. Шымкент: Гылая, 1993. 328 с.

32. Нахушев А. М. Задачи со смещением для уравнений в частных производных. М.: Наука, 2006. 287 с.

33. Сабитов К. Б. К теории уравнений смешанного типа. М.: Физматлит, 2014. 301 с.

34. Сабитов К. Б. Прямые и обратные задачи для уравнений параболо-гиперболического muna. Уфа: Гилем, 2015. 240 с.

35. Нахушев А. М. Новая краевая задача для одного вырождающегося гиперболического уравнения // Докл. АН СССР, 1969. Т. 187, № 4. С. 736-739. 


\title{
MSC: $35 \mathrm{~L} 80,35 \mathrm{~L} 81$
}

\section{The problem with shift for a degenerate hyperbolic equation of the first kind}

\author{
(C) Zh. A. Balkizov
}

Institute of Applied Mathematics and Automation of Kabardin-Balkar Scientific Centre of RAS,

89 a, Shortanova st., Nal'chik, 360000, Russian Federation.

\begin{abstract}
For a degenerate first-order hyperbolic equation of the second order containing a term with a lower derivative, we study two boundary value problems with an offset that generalize the well-known first and second Darboux problems. Theorems on an existence of the unique regular solution of problems are proved under certain conditions on given functions and parameters included in the formulation of the problems under study. The properties of all regular solutions of the equation under consideration are revealed, which are analogues of the mean value theorems for the wave equation.
\end{abstract}

Keywords: degenerate hyperbolic equations, Goursat problem, Darboux problem, problem with shift, mean value theorem.

Received: 20 ${ }^{\text {th }}$ April, 2020 / Revised: $12^{\text {th }}$ February, 2021

Accepted: $10^{\text {th }}$ March, $2021 /$ First online: $29^{\text {th }}$ March, 2021

Competing interests. I declare that I have no apparent or potential conflicts of interest related to the publication of this article.

Authors' contributions and responsibilities. I take full responsibility for submitting the final manuscript in print. I approved the final version of the manuscript.

Funding. This research received no specific grant from any funding agency in the public, commercial, or not-for-profit sectors.

\section{References}

1. Samko S. G., Kilbas A. A., Marichev O. I. Fractional Integrals and Derivatives: Theory and Applications. New York, Gordon and Breach, 1993, xxxvi+976 pp.

2. Nakhushev A. M. Drobnoe ischislenie $i$ ego primenenie [Fractional Calculus and Its Applications]. Moscow, Fizmatlit, 2003, 272 pp. (In Russian)

\section{Research Article}

( )(i) The content is published under the terms of the Creative Commons Attribution 4.0 International License (http://creativecommons.org/licenses/by/4.0/)

Please cite this article in press as:

Balkizov Zh. A. The problem with shift for a degenerate hyperbolic equation of the first kind, Vestn. Samar. Gos. Tekhn. Univ., Ser. Fiz.-Mat. Nauki [J. Samara State Tech. Univ., Ser. Phys. Math. Sci.], 2021, vol. 25, no. 1, pp. 21-34. https://doi.org/10.14498/vsgtu1783 (In Russian).

\section{Author's Details:}

Zhiraslan A. Balkizov (10) https://orcid.org/0000-0001-5329-7766

Cand. Phys. \& Math. Sci.; Leading Researcher; Dept. of Mixed Type Equations;

e-mail: giraslan@yandex.ru 
3. Smirnov M. M. Vyrozhdaiushchiesia giperbolicheskie uravneniia [Degenerate Hyperbolic Equations]. Minsk, Vysh. shk., 1977, 160 pp. (In Russian)

4. Bitsadze A. V. Uravneniia smeshannogo tipa [Equations of Mixed Type]. Moscow, USSR Acad. Sci., 1959, 164 pp. (In Russian)

5. Luikov A. V. Application of the methods of thermodynamics of irreversible processes to the investigation of heat and mass transfer, J. Eng. Phys., 1965, vol. 9, no. 3, pp. 189-202. https://doi.org/10.1007/BF00828333.

6. Bitsadze A. V. Nekotorye klassy uravnenii v chastnykh proizvodnykh [Some Classes of Partial Differential Equations]. Moscow, Nauka, 1981, 448 pp. (In Russian)

7. Nakhushev A. M. The Darboux problem for degenerate hyperbolic equations, Differ. Uravn., 1971, vol. 7, no. 1, pp. 49-56 (In Russian).

8. Nakhushev A. M. Uravneniia matematicheskoi biologii [Equations of Mathematical Biology]. Moscow, Vyssh. shk., 1995, 301 pp. (In Russian)

9. Bers L. Mathematical Aspects of Subsonic and Transonic Gas Dynamics, Surveys in Applied Mathematics, vol. 3. New York, John Wiley \& Sons, 1958, xv+278 pp.

10. Frankl' F. I. Izbrannye trudy po gazovoi dinamike [Selected Works in Gas Dynamics]. Moscow, Nauka, 1973, 711 pp. (In Russian)

11. Gellerstedt S. Sur une équation linéaire aux dérivées partielles de type mixte, Ark. Mat. Astron. Fys. A, 1937, vol. 25, no. 29, pp. 1-23.

12. Kal'menov T. Sh. A criterion for the uniqueness of the solution of the Darboux problem for a certain degenerate hyperbolic equation, Differ. Uravn., 1971, vol. 7, no. 1, pp. 178-181 (In Russian).

13. Kal'menov T. Sh. The Darboux problem for a certain degenerate equation, Differ. Uravn., 1974, vol. 10, no. 1, pp. 59-68 (In Russian).

14. Kal'menov T. Sh. A criterion for the continuity of the solution of the Goursat problem for a certain degenerate equation, Differ. Uravn., 1972, vol. 8, no. 1, pp. 41-54 (In Russian).

15. Balkizov Zh. A. The boundary value problem for a degenerate hyperbolic equation in the area, Izv. Vuz. Severo-Kavkaz. Region. Ser. Estestv. Nauki, 2016, no. 1(189), pp. 5-10 (In Russian). https://doi.org/10.18522/0321-3005-2016-1-5-10.

16. Balkizov Zh. A. The first boundary value problem for a degenerate hyperbolic equation, Vladikavkaz. Mat. Zh., 2016, vol. 18, no. 2, pp. 19-30 (In Russian).

17. Kirichenko S. V. A mixed problem with integral condition for a degenerative equation of the hyperbolic type, Vestn. Samar. Gos. Univ., Estestvennonauchn. Ser., 2011, vol.17, no.8, pp. 29-36 (In Russian). https://doi.org/10.18287/2541-7525-2011-17-8-29-36.

18. Repin O. A., Kumykova S. K. On a problem with generalized operators of fractional differentiation for a degenerated inside a domain hyperbolic equation, Vestn. Samar. Gos. Univ., Estestvennonauchn. Ser., 2012, vol.18, no.9, pp. 52-60 (In Russian). https://doi.org/ 10.18287/2541-7525-2012-18-9-52-60.

19. Repin O. A., Kumykova S. K. A boundary-value problem with shift for a hyperbolic equation degenerate in the interior of a region, Vestn. Samar. Gos. Tekhn. Univ., Ser. Fiz.-Mat. Nauki [J. Samara State Tech. Univ., Ser. Phys. Math. Sci.], 2014, no. 1(34), pp. 37-47 (In Russian). https://doi.org/10.14498/vsgtu1280.

20. Repin O. A., Kumykova S. K. On a class of nonlocal problems for hyperbolic equations with degeneration of type and order, Vestn. Samar. Gos. Tekhn. Univ., Ser. Fiz.-Mat. Nauki [J. Samara State Tech. Univ., Ser. Phys. Math. Sci.], 2014, no.4(37), pp. 22-32 (In Russian). https://doi.org/10.14498/vsgtu1348.

21. Ehrgashev T. G. Generalized solutions of the degenerate hyperbolic equation of the second kind with a spectral parameter, Vestn. Tomsk. Gos. Univ. Mat. Mekh., 2017, no. 46, pp. 4149 (In Russian). https://doi.org/10.17223/19988621/46/6.

22. Makaova R. Kh. A boundary value problem for a third order hyperbolic equation with degeneration of order inside the domain, Vestn. Samar. Gos. Tekhn. Univ., Ser. Fiz.-Mat. Nauki [J. Samara State Tech. Univ., Ser. Phys. Math. Sci.], 2017, vol. 21, no. 4, pp. 651-664 (In Russian). https://doi.org/10.14498/vsgtu1574. 
23. Sabitov K. B., Zaitseva N. V. Initial-boundary value problem for hyperbolic equation with singular coefficient and integral condition of second kind, Lobachevskii J. Math., 2018, vol. 39, no. 9, pp. 1419-1427. https://doi.org/10.1134/S1995080218090299.

24. Sabitov K. B., Zaitseva N. V. The second initial-boundary value problem for a $B$-hyperbolic equation, Russian Math. (Iz. VUZ), 2019, vol.63, no.10, pp. 66-76. https://doi.org/ 10.3103/S1066369X19100086.

25. Sabitov K. B., Sidorov S. N. Initial-boundary-value problem for inhomogeneous degenerate equations of mixed parabolic-hyperbolic type, J. Math. Sci., 2019, vol.236, no. 6, pp. 603640. https://doi.org/10.1007/s10958-018-4136-y.

26. Urinov A. K., Okboev A. B. Modified Cauchy problem for one degenerate hyperbolic equation of the second kind, Ukr. Math. J., 2020, vol.72, no.1, pp. 114-135. https://doi.org/ 10.1007/s11253-020-01766-1.

27. Makaova R. Kh. Boundary-value problem for a third-order hyperbolic equation that is degenerate inside a domain and contains the Aller operator in the principal part, J. Math. Sci., 2020, vol. 250, pp. 780-787. https://doi.org/10.1007/s10958-020-05043-1.

28. Kozhanov A. I. Initial-boundary value problems for degenerate hyperbolic equations, Sib. Èlektron. Mat. Izv., 2021, vol.18, pp. 43-53 (In Russian). https://doi.org/10.33048/ semi.2021.18.004.

29. Nakhushev A. M. Ob odnom klasse lineinykh kraevykh zadach dlia giperbolicheskogo i smeshannogo tipov uravnenii vtorogo poriadka [On a Class of Linear Boundary Value Problems for Second Order Hyperbolic and Mixed Type Equations]. Nal'chik, El'brus, 1992, 155 pp. (In Russian)

30. Репин О. А. Краевые задачи со смещением для уравнений гиперболического и смешанного типов. Самара, Саратовск. гос. унив., Самарск. фил., 1992, 164 с.

31. Кальменов Т. Ш. Краевые задачи для линейных уравнений в частных производных гиперболического типа. Шымкент, Гылая, 1993, 328 с.

32. Nakhushev A. M. Zadachi so smeshcheniem dlia uravnenii v chastnykh proizvodnykh [Problems with Shifts for Partial Differential Equations]. Moscow, Nauka, 2006, 287 pp. (In Russian)

33. Sabitov K. B. K teorii uravnenii smeshannogo tipa [On the Theory of Mixed-Type Equations]. Moscow, Fizmatlit, 2014, 301 pp. (In Russian)

34. Sabitov K. B. Priamye $i$ obratnye zadachi dlia uravnenii parabolo-giperbolicheskogo tipa [Direct and Inverse Problems for Equations of Mixed Parabolic-Hyperbolic Type]. Ufa, Gilem, 2015, 240 pp. (In Russian)

35. Nakhushev A. M. A new boundary value problem for a degenerate hyperbolic equation, Sov. Math., Dokl., 1969, vol.10, no.4, pp. 935-938. 